\title{
ESQUEMAS DE PAGO DE LECHE EN EL SUR DE CHILE: PRECIO MÍNIMO Y MÁXIMO A PRODUCTOR ${ }^{1}$
}

\author{
MILK PAYMENT SYSTEM IN SOUTHERN CHILE: \\ MINIMUM AND MAXIMUM MILK PRICE TO FARMER
}

\author{
Bernardo L. Carrillo L. ${ }^{2}$; Daniela M. Rosas B. ${ }^{3}$; Víctor H. Moreira L. ${ }^{4}$; Juan R. Lerdon F. ${ }^{5}$
}

\section{RESUMEN}

El objetivo del presente trabajo fue analizar los esquemas de pago de leche en cuatro industrias lácteas del sur de Chile. Se recopilaron todos los esquemas desde enero del 1998 hasta junio del 2004, y se utilizaron para estimar los precios máximos y mínimos por litro de leche. Se estimó el impacto relativo de cada variable en el precio final, tanto en el máximo como en el mínimo. En general, las bonificaciones y descuentos por litro de leche presentaron diferencias significativas entre los esquemas de pago y el tiempo de evaluación. Las variables de mayor impacto relativo en el precio final máximo por litro de leche fueron el precio base y volumen de entrega de leche; por otra parte, en el precio final mínimo fueron el recuento de células somáticas y el contenido de unidades formadoras de colonias de MO.

Palabras clave: Precio de leche, precio máximo, precio mínimo, sur de Chile.

\begin{abstract}
The goal of this paper is to analyze the milk payment system of four milk industries of the Los Ríos and Los Lagos regions, located in southern Chile. The collected data were the milk payment systems from January 1998 until June 2004. The collected information allowed the analysis of minimum and maximum estimated milk prices, and the estimation of relative impact of each variable in the milk price. The main results evidence a significant influence of both the milk payment systems and time in the milk price. The most significant variables for the estimated maximum milk price are the base price and the total milk delivered to the industry; and for the estimated minimum milk price are the somatic cell count and colony-forming units.
\end{abstract}

Key words: Milk price, maximum price, minimum price, southern Chile.

\section{INTRODUCCIÓN}

El precio que reciben los productores por su leche depende de las características del mercado. La dinámica del precio se manifiesta a través de esquemas de pago, definidos por las empresas en consideración a características de la leche requerida para elaborar productos. En Chile los esquemas establecen bonificaciones o descuentos de acuerdo a requisitos, lo que ha contribuido a mejorar la calidad de la leche y las características de los productos lácteos. Los esquemas son cada vez más exigentes, y han experimentado constantes modificaciones (Carrillo y Vidal, 2002).

Hasta 1995 la compra de leche a productores estuvo regulada por el Decreto 271, que estableció

1 Este trabajo fue financiado parcialmente por el Fondo de Fomento al Desarrollo Científico y Tecnológico (FONDEF) de la Comisión Nacional de Investigación Científica y Tecnológica (CONICYT) de Chile, bajo el código DO3i-1151.

2 Universidad Austral de Chile, Facultad de Ciencias Agrarias. Independencia 641, casilla 567, Valdivia, Chile.

E-mail: bcarrill@uach.cl

3 Universidad Austral de Chile, Facultad de Ciencias Agrarias. Independencia 641, casilla 567, Valdivia, Chile. E-mail: danielarosas@todoagro.cl

4 Universidad Austral de Chile, Facultad de Ciencias Agrarias. Independencia 641, casilla 567, Valdivia, Chile. E-mail: vmoreira@uach.cl.

5 Universidad Austral de Chile, Facultad de Ciencias Agrarias. Independencia 641, casilla 567, Valdivia, Chile. Email: jlerdon@uach.cl 
por primera vez en Chile un sistema de clasificación (Carrillo y Vidal, 2002). De esta manera las empresas comenzaron a estimular a los productores para producir leche de mejor calidad (Kruze, 1999). Carrillo y Vidal (2002) indican que el Decreto 271 perdió fuerza legal, desde que las industrias implementaron esquemas de pago.

Engler y Nahuelhual (2003) señalan que sobre un precio base se aplican diversas bonificaciones o descuentos según una serie de parámetros. Los esquemas pueden verse afectados por períodos de recesión y de alto crecimiento económico (Vargas, 2000). En algunos países las bases para pagar la leche las determinaba el Estado, en otros se determinan entre la industria y los productores (International Dairy Federation, IDF/FIL 1999). Las empresas lácteas de Chile han incluido parámetros similares a los establecidos en otros países del mundo. El aumento de las exigencias ha obligado a los productores a adaptarse a éstas o dejar de recibir ingresos económicos (Carrillo y Vidal, 2002).

En general, los esquemas consideran un precio base, sobre el cual se fijan bonificaciones o descuentos; un precio base excedente para litros no cubiertos por producción invernal (Carrillo y Vidal, 2002); una bonificación o descuento según el recuento de células somáticas $(r c s)$ y una bonificación o descuento según el recuento bacteriológico ( $u f c / \mathrm{ml}$ ) (Vargas, 2000); un descuento si existe presencia de inhibidores (Kruze, 1999); una bonificación o descuento según contenido de grasa y según contenido de proteína (Carrillo y Vidal, 2002); una bonificación por volumen; una bonificación por estacionalidad; una bonificación por producción invernal y una bonificación por leche fría (Pinto et al., 2002).

Sin embargo, se desconoce el mecanismo que se sigue para establecer las variables y los niveles de cada una de éstas. De allí la importancia de analizar los distintos esquemas de pago de leche y evaluar su comportamiento a través del tiempo. El objetivo del presente trabajo fue analizar la evolución de cuatro esquemas de pago de leche de industrias ubicadas en el sur de Chile, en base a una recopilación de éstos entre 1998 y el 2004. Posteriormente se realizó una simulación del precio máximo y mínimo por litro de leche a productor. Finalmente, se caracterizó la evolución de los esquemas y se determinó el impacto relativo de cada variable en el precio final.

\section{MATERIAL Y MÉTODOS}

Se utilizaron los esquemas de pago de cuatro empresas lácteas, publicados en diarios, y la información que aparece en circulares que se distribuye a productores. Los esquemas se codificaron con números correlativos del I al IV. Se completó una serie de tiempo de seis años y medio (enero 1998-junio 2004). De cada esquema se analizaron dos precios: el precio máximo posible de alcanzar, determinado mediante el reconocimiento de las máximas bonificaciones que pudiese obtener un productor por cada variable, y el precio mínimo, el que se obtuvo mediante el reconocimiento de los descuentos que pudiese obtener el productor. Para efectuar la estimación se utilizaron dos esquemas por año, el de junio y diciembre, meses que representan una situación promedio de los períodos otoñoinvierno y primavera-verano; además, coinciden con la mínima y la máxima recepción de leche.

Las variables analizadas fueron, principalmente: volumen de leche, leche excedente, frío, materia grasa, proteína, unidades formadoras de colonia $(u f c), r c s$, estacionalidad y "bonos". Los bonos para la obtención del precio máximo correspondieron a: bonificación por producción invernal, "pago diferido" (un esquema); en otro se consideraron bonos especiales (entregados en diferentes épocas del año). Para la obtención del precio mínimo, los bonos incluidos corresponden a: producción invernal; para un esquema se agregó bono especial (años 2003 y 2004). Para la evaluación se calculó un precio base promedio, tomando todos los del período (enero 1998-junio 2004). A través de un análisis de varianza se evaluaron las diferencias entre esquemas así como el efecto del tiempo.

\section{RESULTADOS Y DISCUSIÓN}

\section{PRECIO MÁXIMO POR LITRO DE LECHE}

Los esquemas II, III y IV no presentaron diferencias significativas entre sí. El I presentó un precio máximo significativamente superior a los otros tres $(\mathrm{P}<0,05)$, el que fue más alto a partir de diciembre de 1998, aunque en junio de 2004 descendió, llegando a ser similar al IV. El precio máximo obtenido por el IV está subvalorado, ya que cada productor es socio de la empresa, y recibe un precio distinto, dependiendo del volumen de 
leche cubierto por acciones y de la distribución de utilidades de la empresa. Esta bonificación no fue considerada en el análisis por ser parámetro variable entre productores, además de su variación entre los años.

En cuanto a variables que componen el precio máximo, el precio base de los esquemas I y II fue significativamente mayor que el del III y el IV $(\mathrm{P}<0,05)$. Desde junio de 2000 a junio de 2001 se mantuvo en aumento para los cuatro esquemas. Es posible que este aumento sea producto de la disminución de la recepción de la leche en planta durante 1999 y 2000, en relación a 1998, según las cifras de la ODEPA (2001). Al aumentar el precio base se estimula producir más leche. Luego del aumento, desde diciembre de 1998 hasta diciembre de 2001 para el esquema IV, así como en diciembre de 2002 el I, II y III presentaron una disminución, pudiendo haber influido en esto la baja del precio internacional de la leche en polvo. En los meses de primavera de 2003 las industrias no redujeron este precio, manteniendo el de invierno; tampoco fueron castigados los excedentes estacionales, por lo que los precios de primavera fueron similares a los de invierno.

En relación al volumen de leche recepcionada, los esquemas I y IV mostraron bonificaciones significativamente menores que el III $(\mathrm{P}<0,05)$. Los cuatro esquemas, desde junio de 1998 a junio de 2000, mantuvieron su bonificación sin fluctuaciones. A partir de diciembre de 2000 el I, II y IV aumentaron la bonificación notoriamente. A excepción del III, a partir del segundo semestre de 2001, se produce un descenso de ésta. El alza por esta bonificación de la empresa III, mantenida hasta junio de 2002, se explicaría por la disminución en la recepción de leche en ésta, que venía descendiendo desde 1999. Al aumentar la bonificación por volumen, se incentiva el aumento de la productividad y el aumento de la recepción de leche en la industria (Esnaola y Amunátegui, 2002).

$\mathrm{Al}$ analizar la variable "bonos", los esquemas I y II fueron significativamente menores que el III y IV $(\mathrm{P}<0,05)$. El I sólo registra bonificaciones por producción invernal en 1998 y el 2000, y el II sólo registra estas bonificaciones a partir de 2003. El III registra bonificaciones por producción invernal entre 1998 y 1999, posteriormente no registra bonificación, volviendo a aparecer en junio de 2002, período a partir del cual se incluye bono por pago diferido. El IV registra bonificaciones por producción invernal durante todo el período evaluado, en el 2003 se suma un bono especial, sin indicarse detalles de este incentivo.

En lo que respecta a la variable ufc, el esquema I entregó una bonificación significativamente mayor que el II, III y IV $(\mathrm{P}<0,05)$. El IV a su vez entregó una bonificación superior al II y III. El I hasta 1999 entregó la máxima bonificación a leches con recuentos $\leq 100 \mathrm{mil} \mathrm{ufc/ml}$; después con recuentos $\leq 30 \mathrm{mil} \mathrm{ufc} / \mathrm{ml}$. El II y III entregaron la máxima bonificación con recuento $\leq 30 \mathrm{mil} \mathrm{ufc/ml}$. El IV hasta 1999 entregó la mejor bonificación con recuento $\leq 50 \mathrm{mil} \mathrm{ufc} / \mathrm{ml}$, posteriormente $\leq 20 \mathrm{mil} \mathrm{ufc} / \mathrm{ml}$. A nivel internacional, la mejor leche debe alcanzar niveles muy bajos, como por ejemplo en Dinamarca la clase I debía alcanzar recuentos $\leq 30 \mathrm{mil} \mathrm{ufc} / \mathrm{ml}$, lo que coincide con lo exigido por los esquemas I, II y III. Países como Argentina y Bélgica clasificaban dentro de la mejor categoría recuentos $\leq 100$ mil ufc/ $\mathrm{ml}$. Nueva Zelanda presentaba mayores exigencias, clasificando en categoría I sólo leches con recuento $\leq 10 \mathrm{mil} \mathrm{ufc/ml}$ (IDF/FIL 2002).

Para la variable rcs el esquema II mostró un valor significativamente más bajo que el resto $(\mathrm{P}<0,05)$. La máxima bonificación la entregaron el I y IV. Según Philpot y Nickerson (2000), la bonificación más alta corresponde a empresas que elaboran queso, por ser un parámetro directamente relacionado con el rendimiento y calidad de éste, lo que coincide con la mayor bonificación de los esquemas I y IV, industrias que elaboraron una mayor cantidad de queso durante el estudio. En el período evaluado, el esquema I aumentó la exigencia para la primera categoría de $400 \mathrm{mil} \mathrm{a} 300 \mathrm{mil} \mathrm{cel} / \mathrm{ml}$. El II, hasta mediados de 2000 , exigía un recuento $\leq 400 \mathrm{mil} \mathrm{cel} / \mathrm{ml}$, posteriormente $\leq 250 \mathrm{mil} \mathrm{cel} / \mathrm{ml}$. Los esquemas III y IV mantuvieron un recuento $\leq 300$ $\mathrm{mil} \mathrm{cel} / \mathrm{ml} \mathrm{y} \leq 250 \mathrm{mil} \mathrm{cel} / \mathrm{ml}$, respectivamente. A nivel internacional, hasta hace algunos años para la categoría I, Canadá exigía recuentos $\leq 500 \mathrm{mil}$ $\mathrm{cel} / \mathrm{ml}$; Argentina $\leq 200 \mathrm{mil} \mathrm{cel} / \mathrm{ml}$, Nueva Zelanda $\leq 400 \mathrm{mil} \mathrm{cel} / \mathrm{ml}$ y Austria $\leq 250 \mathrm{mil} \mathrm{cel} / \mathrm{ml}$ (IDF/ FIL 2002).

$\mathrm{Al}$ analizar la variable frío, el esquema I presentó los mayores valores de bonificación $(\mathrm{P}<0,05)$. El II y III no presentaron diferencias significativas entre sí, y el IV presentó los menores valores. La industria establece como requisito la compra de leche fría $\left(4{ }^{\circ} \mathrm{C}\right)$, pudiendo alcanzar la máxima bonificación productores propietarios del estanque (Carrillo y Vidal, 2002). Cuando éste es de la industria, la 
bonificación es menor. Países como Canadá, Bélgica, Argentina, Nueva Zelanda, Francia, USA, España y Austria también exigen la refrigeración de la leche en el predio (IDF/FIL 2002).

En relación a la bonificación por estacionalidad, la del esquema IV fue significativamente superior a los otros tres, seguido del II $(\mathrm{P}<0,05)$. La importancia relativa de esta bonificación depende de la estructura del mercado consumidor; si éste requiere de leche fluida o productos frescos, es necesario contar con ofertas constantes de leche (Fundación Chile, 2000). Esto podría explicar la mayor bonificación entregada por el esquema IV, perteneciente a la empresa que elaboró la mayor cantidad de productos frescos y leche fluida durante el período analizado. La industria II fue la segunda en elaboración de leche fluida (ODEPA, 1999, 2000, 2001, 2002, 2003).

La bonificación por proteína del esquema IV fue significativamente superior a la del I, II y III $(\mathrm{P}<0,05)$. El III a partir de junio de 2000, comienza a entregar la bonificación más alta, alcanzando el máximo en junio de 2004. El IV hasta diciembre de 1999 entregó la mayor bonificación; posteriormente sigue un comportamiento similar al III. Coincidiendo con ello la empresa IV elaboró la mayor cantidad de queso durante el período estudiado según ODEPA (1999, 2000, 2001, 2002, 2003).

\section{VALOR RELATIVO DE CADA VARIABLE EN EL PRECIO MÁXIMO}

Los valores utilizados para este cálculo fueron el promedio de cada variable. De la Figura 1 se deduce que la variable que más influyó en el precio máximo fue el precio base, válido para los cuatro esquemas, contribuyendo en 50\% o más. Las variables $u f c$, volumen y rcs mostraron alta participación del precio final para el esquema I. Para el II, después del precio base, le siguieron las variables volumen, $u f c$, estacionalidad y $r c s$. En el III, las variables que contribuyeron en forma importante fueron volumen, "bonos", ufc y rcs. Para el IV, las variables de mayor importancia fueron estacionalidad, volumen, $r c s, u f c$ y bonos.

\section{PRECIO MÍNIMO POR LITRO DE LECHE}

La bonificación mínima por volumen fue significativamente distinta entre los esquemas $(\mathrm{P}<0,05)$. El I presentó la menor bonificación,

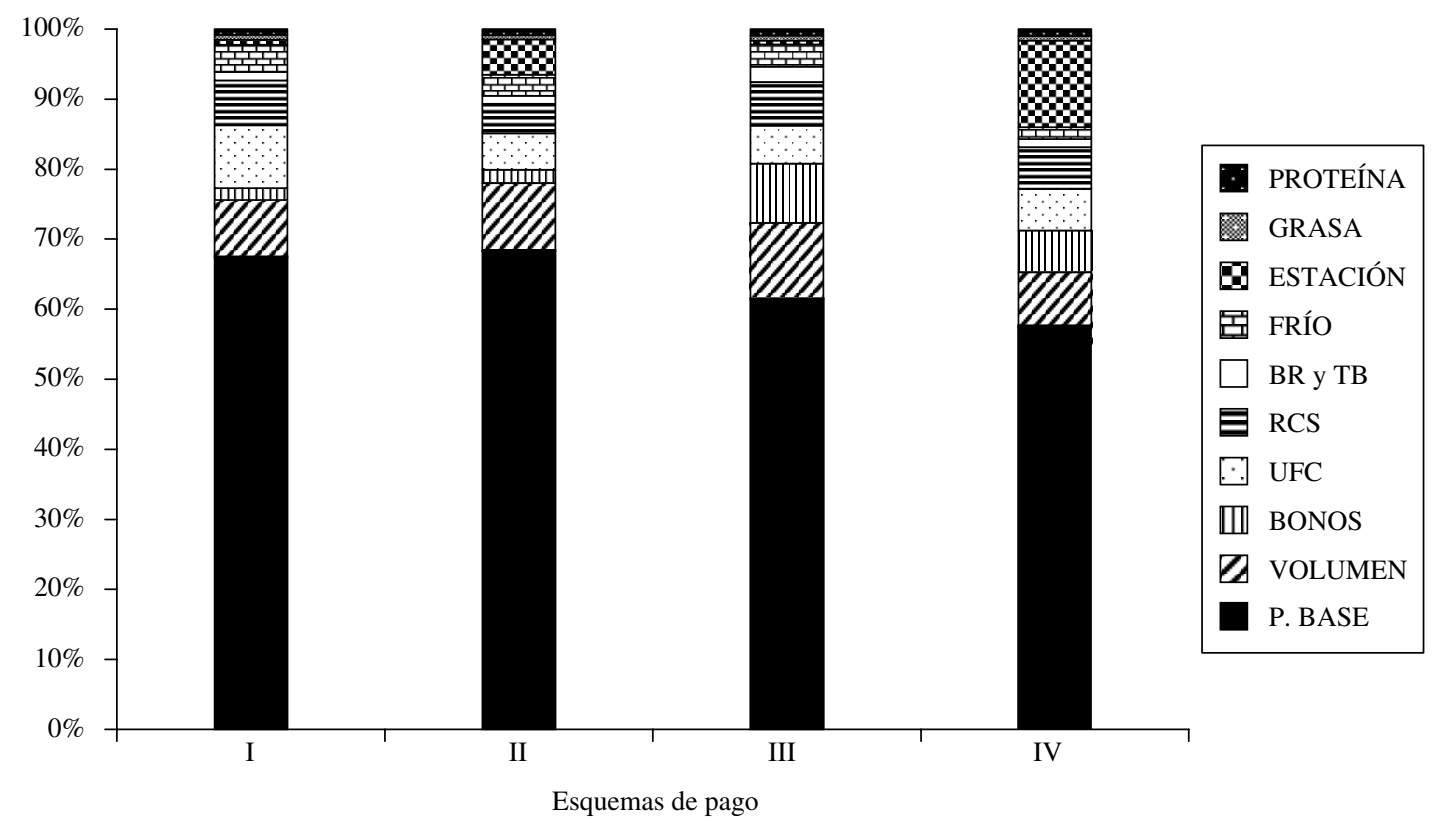

Fuente: Elaboración propia en base a resultados del estudio.

Figura 1. Peso relativo de cada variable en el precio máximo, para cuatro esquemas de pago de leche. 
seguido del II, III y IV. El I sólo en junio de 2004 comienza a bonificar leche del estrato de menor volumen. El III mantuvo la mayor bonificación por más tiempo (diciembre de 1999 a junio de 2002). El aumento de la mínima bonificación por volumen del III, desde diciembre de 1999 a diciembre del 2000, coincidió con la disminución en la recepción de leche a nivel nacional (ODEPA, 2003). El IV, hasta junio de 1999, bonificó a productores que entregaban menores volúmenes de leche; posteriormente fue suspendida, para volver a aplicarse en diciembre de 2002.

La contribución mínima obtenida por "bonos" incluyó a la producción invernal, además en el caso del esquema IV agregó un bono especial entregado en algunos meses de 2003 y 2004. Éstos fueron significativamente distintos en relación al esquema de pago $(\mathrm{P}<0,05)$. El I presentó diferencias significativas con III y IV. El II no presentó diferencias con III, y éste a su vez no presentó diferencias con IV. Los esquemas III y IV son los que presentaron las bonificaciones más altas. Todos los esquemas no presentaron bonificación por la producción invernal entre junio de 2001 y junio de 2002. Según ODEPA (2002), el 2001 el precio de invierno no se diferenció del de primavera, debido a la acumulación de existencias, producto de un error en el que incurrieron algunas industrias al importar volúmenes considerables e innecesarios de leche en polvo a altos precios.

El descuento aplicado según el recuento de $u f c$ fue significativamente distinto entre los esquemas de pago y el tiempo $(\mathrm{P}<0,05)$. El I y III aplicaron descuentos más altos. El I aplicó el mayor descuento por $u f c$ a partir de diciembre de 2000, y el mayor descuento se produjo en junio de 2003. El IV aplicó el menor descuento a lo largo de todo el período; sin embargo, fue el más exigente en cuanto al umbral establecido para el contenido de $u f c$, ya que con un recuento $\geq 200 \mathrm{mil} \mathrm{ufc/ml} \mathrm{comenzaba}$ a descontar.

En cuanto a descuentos aplicados por $r c s$, existieron diferencias significativas entre los esquemas $(\mathrm{P}<0,05)$. El mayor fue aplicado por el IV, aumentando en diciembre de 2001, y acentuándose en junio de 2004 para leche con $\geq 750.000$ cel/ml. Según Philpot y Nickerson (2000), altos $r c s$ provocan degradación enzimática de proteína durante almacenamiento en quesos, lo cual genera problemas de olor y textura. Por su parte, FIL/IDF (2005) y Revelli y Rodríguez (2001) señalan que leche con alto recuento aumenta la actividad proteolítica y lipolítica, disminuye la firmeza de la cuajada, provoca pérdida de caseína y aumento de proteínas solubles, con lo que se obtiene menor rendimiento. Esto podría explicar el mayor descuento establecido por la empresa IV, ya que durante el período evaluado era la principal elaboradora de queso (ODEPA 1999, 2000, 2001, 2002, 2003).

Los esquemas establecen rangos para $r c s$. La empresa I aplicó el máximo descuento a leche con $\geq 800 \mathrm{mil} \mathrm{cel} / \mathrm{ml}$; exigencia que comenzó el segundo semestre de 1999. El II y III aplican el máximo descuento con recuento $\geq 600 \mathrm{mil} \mathrm{cel} / \mathrm{ml}$ $\mathrm{y} \geq 700 \mathrm{mil} \mathrm{cel} / \mathrm{ml}$, respectivamente. Los esquemas II y III comienzan a exigir este recuento el segundo semestre de 1999 y de 2000, respectivamente. A nivel internacional, también existen diferentes categorías. En Dinamarca y Nueva Zelanda leche que contiene $\geq 600 \mathrm{mil} \mathrm{cel} / \mathrm{ml}$ corresponde a la categoría más baja. Para Bélgica y Austria, las exigencias son mayores, quedando dentro de la categoría más baja leche con $\geq 400 \mathrm{mil} \mathrm{cel} / \mathrm{ml}$ (IDF/FIL 2002).

En lo que respecta a la bonificación mínima por concepto de frío, el esquema I entregó ésta hasta diciembre de 1998. A partir de junio de 1999 bonificaron los esquemas I y II productores con estanque propio. El III solo bonificó hasta octubre de 1999 a productores que no tenían estanque propio. El IV bonificó en todo el período evaluado, aunque la bonificación fue inferior cuando el estanque era propiedad de la industria. Hasta hace algunos años en países como Dinamarca, Francia e Irlanda se pagaba un incentivo por enfriamiento de la leche en predio y, en el caso de Francia, el precio de la leche variaba con la propiedad del estanque de frío (Latrille, 1999).

Las diferencias en el descuento por producción de leche excedente fueron significativas con respecto al tiempo $(\mathrm{P}<0,05)$. En diciembre de 2000 , el esquema III no aplicó descuento, probablemente como medida para estimular la producción, que venía disminuyendo por segundo año consecutivo. En diciembre de 2003, ningún esquema aplicó descuento, debido probablemente al alza de los precios internacionales y al mayor costo de importación de la leche en polvo; a lo que se sumó la fuerte caída de la leche entregada en planta el segundo trimestre de 2003. Debido a esto las industrias redujeron solo levemente el precio de invierno y no castigaron el excedente estacional, por lo que los precios de primavera fueron muy similares a los alcanzados en invierno. 
El descuento aplicado por contenido de proteína fue significativamente diferente entre los esquemas y el tiempo $(\mathrm{P}<0,05)$. El III y IV aplicaron descuentos similares y menores que I y II. El III a partir de junio de 2000 aplicó el mayor descuento, similar al aplicado por el IV. Los esquemas I y II son los que menor descuento aplicaron, aunque en junio de 2003 el II igualó el descuento aplicado por el IV.

El descuento aplicado por contenido de materia grasa fue significativamente diferente con respecto al esquema de pago $(\mathrm{P}<0,05)$. El IV aplicó el mayor descuento, el que mantuvo casi todo el período, y el II el menor. El II presentó el menor descuento, coincidiendo con el esquema III, entre diciembre de 2000 y junio de 2002. Posterior a este período, el III aumentó el descuento por este concepto.

La importancia relativa de cada variable en el precio mínimo final se calculó usando el valor promedio de cada variable. La calidad higiénica, la producción de leche excedente, el contenido proteico y la grasa fueron las que más contribuyeron en la obtención del precio mínimo por litro de leche para los cuatro esquemas (Figura 2).

\section{CONCLUSIONES}

$\mathrm{Al}$ analizar la evolución de los cuatro esquemas de pago, se encontraron diferencias entre éstos y entre el período de tiempo evaluado. El esquema I destacó por presentar el precio base y las bonificaciones más altas por $r c s$ y contenido de ufc. En relación a esta última variable, es la misma planta la que presenta el descuento más alto, junto a los menores bonos. El II, presentó el precio base más alto, y el mayor descuento por frío. El III destaca por presentar las mayores bonificaciones por volumen de leche, sanidad del rebaño y bonos; las dos primeras variables fueron las que presentaron los mayores descuentos. El IV presenta las mayores bonificaciones por estacionalidad de producción, proteína y grasa, variables que más el $r c s$ fueron las que presentaron los mayores descuentos.

En promedio, las variables que tuvieron más peso relativo en el precio final máximo por litro de leche fueron el precio base y volumen de entrega de leche. En la obtención del precio final mínimo las variables de mayor peso fueron el $r c s$ y las $u f c$.

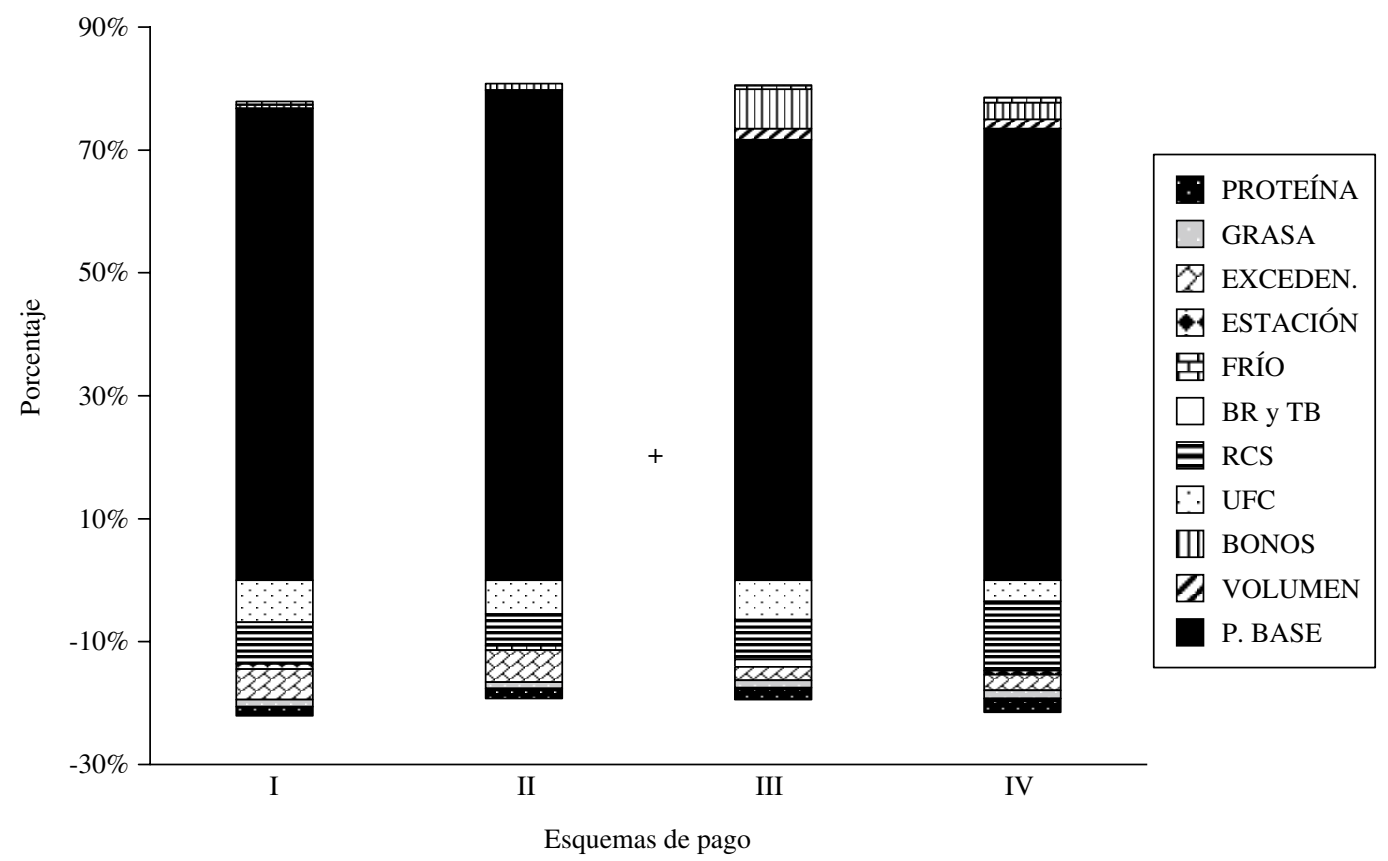

Fuente: Elaboración propia en base a resultados del estudio.

Figura 2. Participación relativa de cada variable en el precio mínimo, para cuatro esquemas de pago de leche. 


\section{LITERATURA CITADA}

CARRILlO, B.; VIDAL, C. 2002. Análisis y aplicación de los esquemas de pago de la leche en la X Región. En: Curso Mercado y gestión económica de la producción de leche. Fundación Chile. Ministerio de Agricultura. Chile, pp. 1-11.

FUNDACIÓN CHILE. 2000. Economía y gestión de la producción lechera. Programa de gestión agropecuaria. Ministerio de Agricultura. Chile, pp. 40-53.

OFICINA DE ESTUDIOS Y POLÍTICAS AGRARIAS (ODEPA). 1999. Boletín de la Leche. Ministerio de Agricultura. Chile. 55 p.

2000. Boletín de la Leche. Ministerio de Agricultura. Departamento Información Agraria. Chile. 55 p. 2001. Boletín de la Leche. Ministerio de Agricultura. Departamento Información Agraria. Chile. 55 p. 2002. Boletín de la Leche. Ministerio de Agricultura. Departamento Información Agraria. Chile. 55 p. 2003. Boletín de la Leche. Ministerio de Agricultura. Departamento Información Agraria. Chile. 55 p.

ESNAOLA, V.; AMUNÁTEGUI, R. 2002. Leche: situación 2002 y perspectiva del sector lácteo. Mercados Agropecuarios 123: 1-4.

ENGLER, A.; NAHUELHUAL, L. 2003. Influencia del mercado internacional de lácteos sobre el precio nacional de la leche: un análisis de cointegración. Agricultura Técnica (63): 416-427.

INTERNATIONAL DAIRY FEDERATION. IDF/FIL. 1999. Fédération international de laitiere-International Dairy Fédération. Payment systems for ex-farm milk results of IDF questionnaire 2399/A:99 (Study group 178). Bulletin (348): 15-42.
2002. Fédération international de laitiere-International Dairy Federation. Payment systems for ex-farm milk results of IDF questionnaire 379/2002.

2005. Economic Consequences of Mastitis. Bull. Int. Dairy Fed. 394, 3-26.

KRUZE, J. 1999. Calidad higiénica de la leche cruda en Chile. En: Latrille, L. (ed.). Avances en Producción Animal. Facultad de Ciencias Agrarias, Universidad Austral de Chile. pp. 107-139.

LATRILLE, L. 1999. Calidad de leche y sistemas de pago. En: Competitividad de la producción lechera nacional. Facultad de Ciencias Agrarias. Universidad Austral de Chile. Tomo II. pp. 259-315.

PHILPOT, N.; NICKERSON, S. 2000. Importancia de la calidad de la leche. Ganando la lucha contra la mastitis. pp. 154-161.

PINTO, M.; CARRILLO, B.; VIDAL, C. 2002. Clasificación de leche por calidad con fines de pago al productor. Los esquemas de pago en Chile. En: I Seminario nacional sobre calidad e inocuidad de la leche y sus derivados. Universidad Simón Bolívar. México. pp. 1-10.

REVELLI, G.; RODRÍGUEZ, C. 2001. Prevalencia de agentes etiológicos causales de mastitis bovina en la zona noroeste de Santa Fe y sur de Santiago del Estero. Respuesta a la sensibilidad antimicrobiana. Revista Tecnología Láctea Latinoamericana (23): 48-53.

VARGAS, G. 2000. Economía y gestión de la producción lechera. Programa de gestión agropecuaria. Fundación Chile. Ministerio de Agricultura. 65 p.

2001. Cambio estructural en el sector lechero chileno: Potencial exportador y desafíos. Ciencia e Investigación Agraria. 28 (3): 117-129. 
